\title{
A High-Altitude Balloon Platform for Space Life Sciences Education
}

\begin{abstract}
Jordan McKaig ${ }^{1}$, Tristan Caro ${ }^{2}$, Alex Hyer $^{3}$, Elizabeth Delgadillo Talburt ${ }^{4}$, Sonali Verma ${ }^{5}$, Kaixin Cui ${ }^{6}$, Anna-Sophia Boguraev Molly Heit ${ }^{8}$, Aimee Johnson ${ }^{9}$, Emily Johnson ${ }^{10}$, Andrew Jong ${ }^{11}$, Brooke Shepard ${ }^{12}$, Jamie Stankiewiz ${ }^{13}$, Nhung Tran $^{14}$, Jon Rask ${ }^{15}$

${ }^{1}$ KBR, NASA Ames Research Center, Moffett Field, CA; 2 Interdisciplinary Quantitative Biology, BioFrontiers Institute, University of Colorado, Boulder, CO: ${ }^{3}$ Department of Biological Sciences, University of Southern California, Los Angeles, CA: ${ }^{4}$ College of Aeronautics, Embry-Riddle Aeronautical University, Daytona Beach, FL; ${ }^{5}$ Blue Marble Space Institute of Sciences, NASA Ames Research Center, Moffett Field, CA; ${ }^{6}$ Pathways Program, NASA Johnson Space Center, Houston, TX; ${ }^{7}$ Department of Molecular Biophysics and Biochemistry, Yale University, New Haven, CT; ${ }^{8}$ Department of Biochemistry, Binghamton University, State University of New York, Binghamton, NY; ${ }^{9}$ Department of Epidemiology, Geisel School of Medicine at Dartmouth, Hanover, $\mathrm{NH} ;{ }^{10}$ Department of Biological Sciences, Kent State University, Kent, OH; "11Department of Computer Science, San José State University, San Jose, CA; ${ }^{12}$ Department of Biology, Oakland University, Rochester, MI; ${ }^{13}$ Department of Molecular \& Cell Biology, University of California, Berkeley, Berkeley, CA; ${ }^{14}$ Division of Biological Sciences, University of California, San Diego, San Diego, CA; ${ }^{15}$ Office of the Center Director, NASA Ames Research Center, Moffett Field, CA
\end{abstract}

Abstract

High-altitude balloons (HABs) present a valuable and cost-effective tool for educators and students to access the conditions that are analogous to space and extraterrestrial environments in the Earth's upper atmosphere. Historically, HABs have been used for meteorological measurements, observation, sampling of aerosols, and exposure of samples to upper atmosphere environments. The Earth's stratosphere allows researchers access to a unique combination of wideband solar radiation, extreme cold, rarefied air, low humidity, and acute ionizing radiation-conditions that are relevant to space biology research. Here, we describe a reproducible payload for a HAB mission that can be constructed, launched, and retrieved for about $\$ 3,000$. This general standard operating procedure can be used by educators, community scientists, and research teams working with limited resources.

Keywords

aerobiology $\bullet$ high-altitude ballooning $\bullet$ citizen science

\section{INTRODUCTION}

High-altitude balloons (HABs) have long been used for experimental access to both the lower and upper atmosphere, including the troposphere and stratosphere. There are various methods for accessing the stratosphere, including meteorological rockets (Imshenetsky et al., 1976), fixed-wing aircraft (Smith et al., 2009), mountaintop observatories (Smith et al., 2011), and high-altitude weather balloons (Bryan et al., 2014); however, HABs are unmatched in their affordability, versatility, and high payload-to-vehicle-mass ratio. In addition, payloads can be often reused. Larger HABs, commonly referred to as scientific balloons, can carry payloads up to $3,600 \mathrm{~kg}$ while staying aloft for weeks or months. At the same time, smaller HABs, commonly referred to as meteorological balloons, typically carry payloads weighing a few kilograms; these may be launched by hand, making them suitable for amateur use. Both types of HABs can rise into the stratosphere to reach the altitudes between 17 and $35 \mathrm{~km}$ above sea level (Smith \& Sowa, 2017).
HABs have provided a useful platform for scientific investigations for over a century. Even as early as the late 18th century, scientists were using balloons to collect data from unprecedented altitudes. Balloon-based experiments aided in early studies of cosmic ray distributions throughout the atmosphere and allowed for the first direct measurements of the stratosphere (Yajima et al., 2004). Since then, HABs' utility has flourished and they have become integral to astrophysical, planetary, and climate studies, including radiation investigations in near-space environments (Lawrence et al., 2018), measurement of electric fields within the stratosphere (Gurubaran et al., 2017), and surveillance of atmospheric ozone levels (Hofmann et al., 1987).

Despite their ubiquity in atmospheric and Earth science research techniques, HABs have only recently been regularly utilized for aerobiological research (Smith \& Sowa, 2017). Access to the stratosphere yields a valuable opportunity for researchers to explore an extreme environment that

${ }^{\dagger}$ Corresponding author: Tristan Caro

E-mail: tristan.caro@colorado.edu 


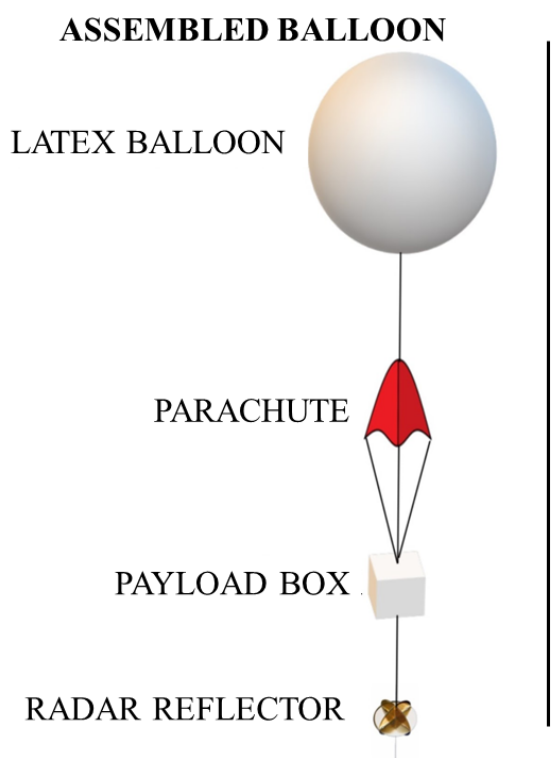

PAYLOAD BOX DETAILS

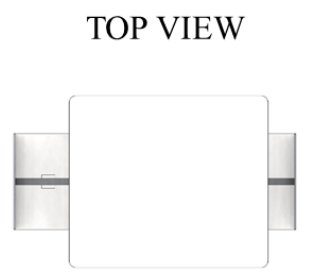

FRONT VIEW

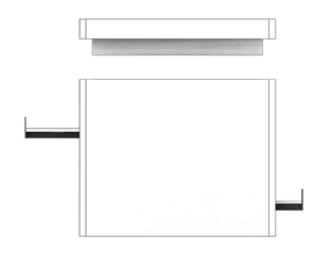

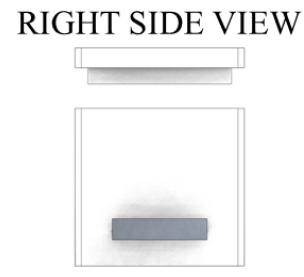

ORTHOGRAPHIC PROJECTION

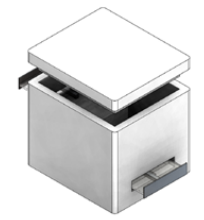

Figure 1. HAB assembled design (left) showing the balloon, parachute, payload box, and radar reflector strung together; payload box design (right) with top view, left view, front view, and exploded orthographic projection.

experiences a combination of wideband solar radiation, extreme cold, rarefied air, low humidity, and acute ionizing radiation. There, stratospheric conditions are analogous to the environmental conditions on the surface of Mars, which experiences a similar cytotoxic ultraviolet (UV) flux, low pressure, and low temperature (Kaplan 1988; Smith 2013). One such study employed a HAB to expose extremophilic yeast to the stratosphere. In this study, the conditions of the upper atmosphere were considered to be of astrobiological relevance as a Mars analog environment (Pulschen et al., 2018).

Meteorological balloons present a valuable and costeffective tool for educators, students, and researchers to access the conditions that are analogous to space and extraterrestrial environments, by the merit of their relatively low cost, ease of retrieval, payload reusability, ability to provide in-situ atmospheric observations, and short preparation time (Yajima et al., 2004). Despite the high frequency of amateur $\mathrm{HAB}$ launches and their ubiquity in atmospheric research, there are few established standard operating procedures for researchers and community scientists to conduct HAB-based experiments.

In this article, we describe a generalizable meteorological balloon payload and outline a standard protocol for HAB missions that can serve as a platform for high-altitude experiments. Our payload was optimized for aerosol sampling and exposure of biological samples; however, the modular nature of the payload design allows for the integration of many different types of experiments and analyses. A single $\mathrm{HAB}$ payload can be assembled in as little as a few weeks with a budget of about $\$ 3,000$. We designed, constructed, and launched two balloons, designated as SHAB-1 and SHAB2 , and successfully obtained relevant meteorological data, including temperature, pressure, and altitude. The methods developed from this project can be applied to future $\mathrm{HAB}$ launches for both educational and research purposes.

\section{METHODS}

\section{Launch Vehicle}

The HAB is composed of a $1.5 \mathrm{~kg}$ latex balloon (High Altitude Science), a $1.5 \mathrm{~m}$ diameter circular parachute (High Altitude Science) carrying two custom-built payloads, and a radar reflector (constructed with aluminum reflective tape and cardboard). These items were attached in series to a paracord Flightline. The diagram is shown in Figure 1.

\section{Payload Description}

The payload carried by the HABs consisted of two biological sample trays (BSTs), which can be used for the collection of microbial material in laminar airflow or exposure of organisms adhered to the coupons. The main payload also carried environmental sensors measuring ambient temperature, atmospheric pressure, and payload velocity; camera (GoPro Hero 5); and an embedded flight computer. A computer-aided design (CAD) of the assembled payload is shown in Figure 1. The flight computer system was controlled by an Arduino Uno based on the ATmega328P chip wired as depicted in 


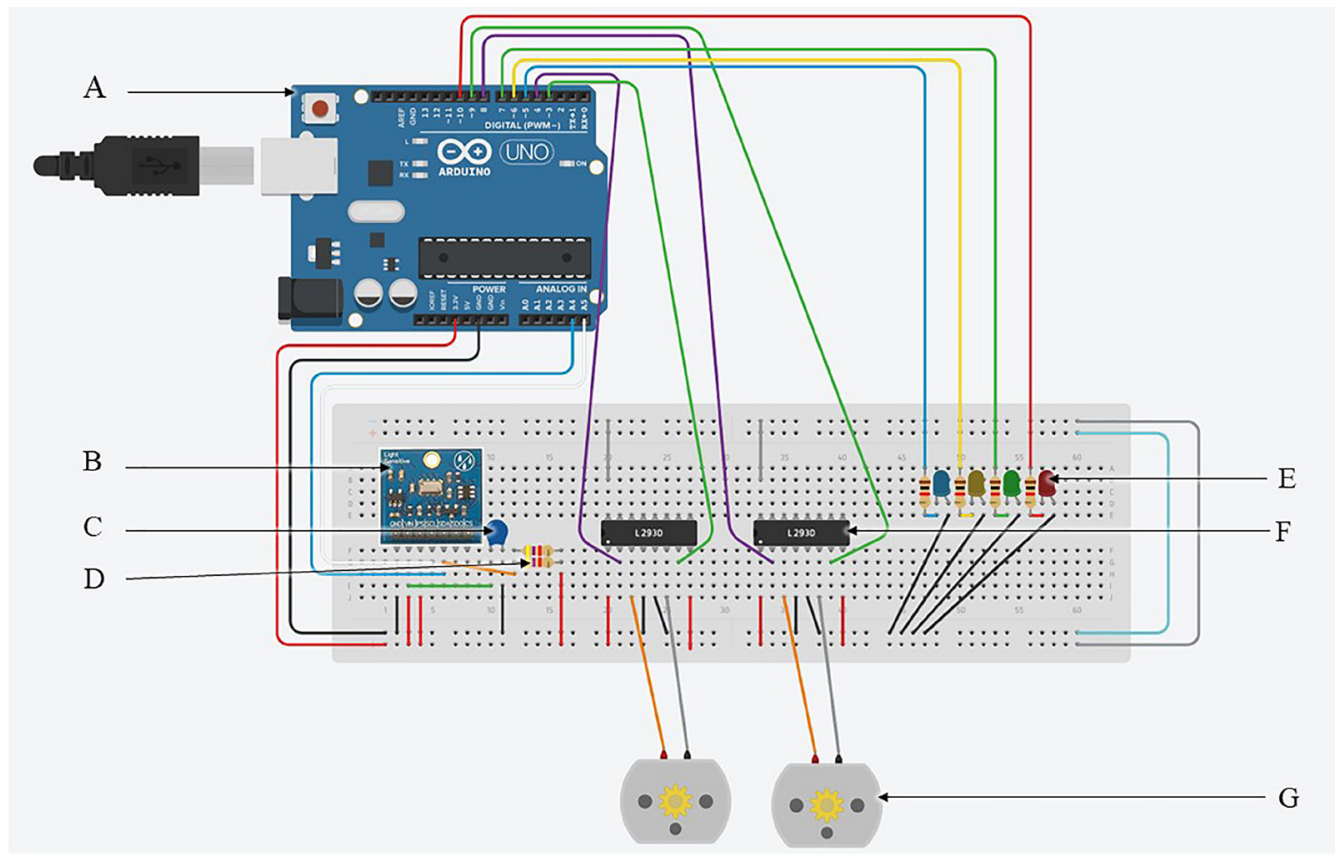

Figure 2. Circuit wiring schematic showing (A) Arduino Uno microcontroller; (B) altimeter module MS5607; (C) $100 \mathrm{nF}$ capacitor; (D) two 4.7 $\mathrm{k} \Omega$ resistors; (E) four colored LEDs, each connected in series with a $1 \mathrm{k} \Omega$ resistor; (F) two L293D H-bridges; and (G) Actuonix L12-R micro linear servos. This circuit controls the flight computer system, such as sensors and actuation mechanisms within the payload.

Figure 2. An Altimeter Module MS5607 (Parallax) was used to assess ambient pressure, temperature, and altitude. The data logging system used a microSD card and a real-time clock for synchronization. These system communication protocols include serial peripheral interface (SPI) and interintegrated circuit (I2C), which are dependent on the sensor. More information about the software used on the flight can be found in our online code repository: https://github.com/ SLSTP-2018/shab.

To track the HAB in-flight, an MT-AIO (Byonics) module using a GPS5HAOEM GPS transmitted telemetry data at a frequency of $144.390 \mathrm{MHz}$ with audio frequency-shift keying (AFSK) at 1,200 baud was used. AFSK packets were received by ground-based Automatic Packet Reporting System (APRS) digipeaters and relayed to a publicly available website (aprs.fi) for real-time position tracking. We also directly received and demodulated AFSK packets using an RTL-SDR USB dongle connected to a personal computer running open-source software-defined radio (SDR), GQRX (gqrx.dk). GQRX SDR includes an AFSK1200 decoder, which prints demodulated APRS telemetry packets. APRS was transmitted through an externally mounted half-wave V6 dipole antenna on path WIDE2-1. APRS transmissions were tagged with an appropriate FCC-issued amateur call sign. For all electronic components, consumer-grade lithium-ion batteries were used, as they are less likely to be affected by cold temperatures and are lighter than alkaline batteries.

\section{Payload Operations}

Sample collection at altitude was accomplished with BSTs that were attached to two linear servos (Actuonix L12-R; $100 \mathrm{~mm}$, 210:1, $6 \mathrm{~V}$ ). The servos were used to move the BSTs from the shielded interior of the payload box (launch configuration) to the outside of the payload box (exposed sampling configuration) at target altitudes of 10,668 and $27,432 \mathrm{~m}$ above sea level. The altitude of the HAB was determined by an onboard altimeter, labeled "B" in Figure 2. When the HAB's altimeter measured the first target altitude, one linear servo actuated a BST to the exterior of the payload, exposing the tray and allowing for atmospheric microbial sampling. A realtime clock tracked exposure time and retracted the BSTs after the programmed exposure time (900 s) had passed. When the second target altitude was reached, the process was repeated with the second linear servo and BST.

Extension and retraction of each BST were achieved using an L293D H-bridge, labeled "F" in Figure 2. The H-bridges reverse the polarity of the voltage delivered to the linear servos, serving as a switch to extend and retract the linear servos. For visual logging of the extension and retraction of the BSTs, a GoPro HERO5 was attached to the exterior of the payload and programmed to capture photos every $30 \mathrm{~s}$ for the duration of the flight.

As a preflight check of the payload hardware operations, four LEDs, labeled "E" in Figure 2, were added to the circuit to indicate errors in the hardware. The four colors corresponded 


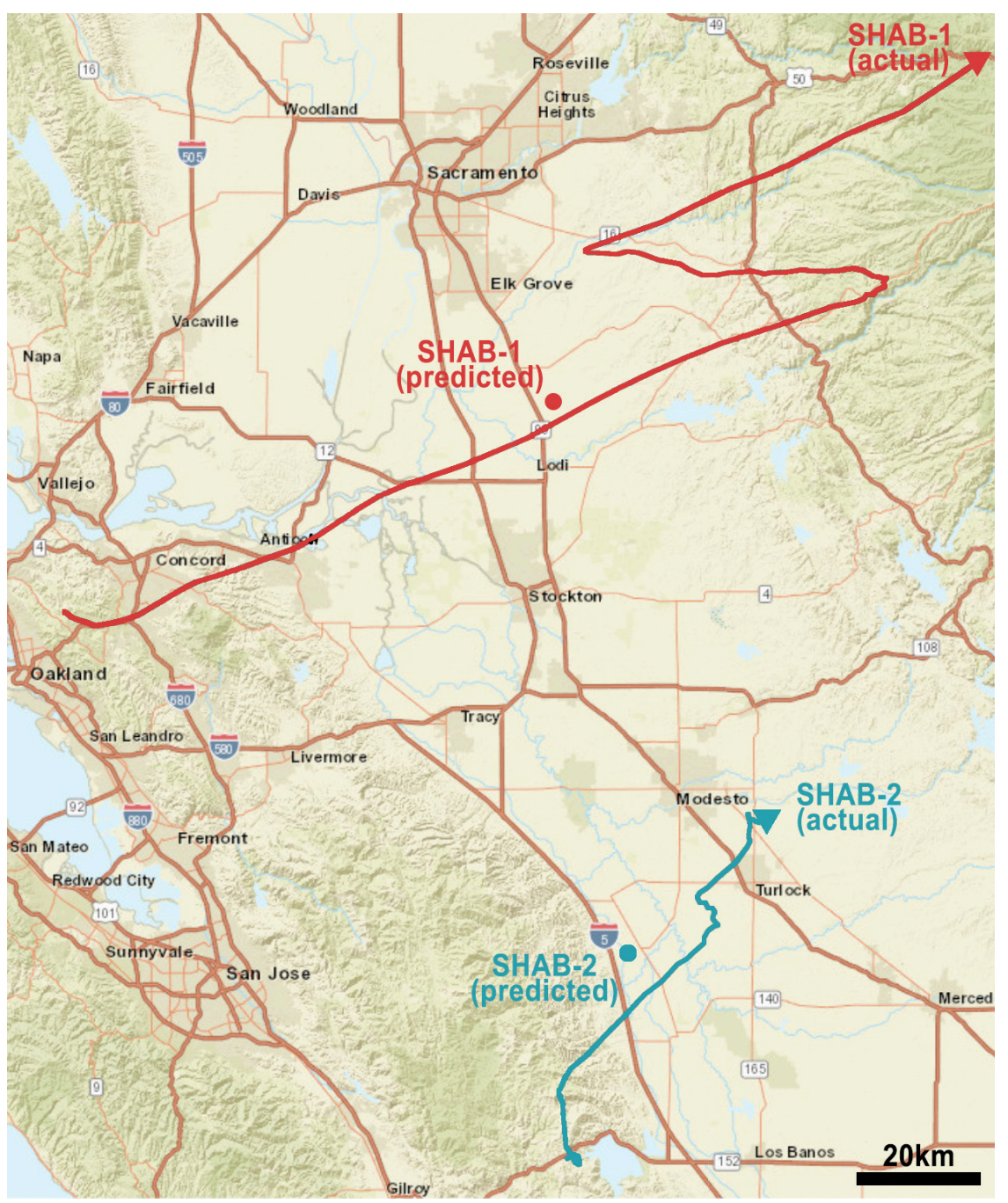

Figure 3. Flight trajectories for SHAB-1 (red) and SHAB-2 (blue), as reported by APRS telemetry. CUSF- predicted landing zones (circle) are compared to actual landing sites (triangle). (Map: ArcGIS).

to different errors in the wiring of specific components in the circuit.

Passive exposure of biological samples to high altitudes required no specialized hardware, except for an OptiCell Cell Culture Chamber (Cat\#: 392-0603) that contained media and the organisms, which was taped to the side of the payload just before launch. Postflight analysis of the organisms required recovery of the samples.

\section{Flight Planning}

HAB flight planning was conducted using the online ASTRA High Altitude Balloon flight prediction software (http://astraplanner.soton.ac.uk/). The software accepts parameters of balloon mass, gas, current weather conditions, and parachute type to run up to 400 discrete flight simulations. ASTRA generates a map of possible flight paths and predicted landing zones, allowing balloon teams to determine optimal launch locations. Desired neck-lift (i.e., the vertical force due to the lift of helium) was calculated using the CUSF Flight predictor tool (http://predict.habhub.org/), which also predicts landing zones based on flight parameters and weather information. Flight trajectories, both actual and predicted, for both HAB flights are displayed in Figure 3.

\section{Flight Software}

A consumer-grade microcontroller (Arduino Uno) was used as an embedded flight computer. Each hardware component was tested for functionality, and a color-coordinated LED was 
lit if the hardware was dysfunctional. The linear actuators were tested before sterilization by extending and retracting each BST to ensure a successful deployment. The BSTs are shown in Figure 4. At 1-s intervals during flight, the barometric pressure sensor and thermometer collected temperature and pressure data, which were used to calculate altitude (Figure 5). In particular, we corrected the pressure reading for the temperature and compared it to an altitude table of known pressures per altitude to calculate the current altitude. This

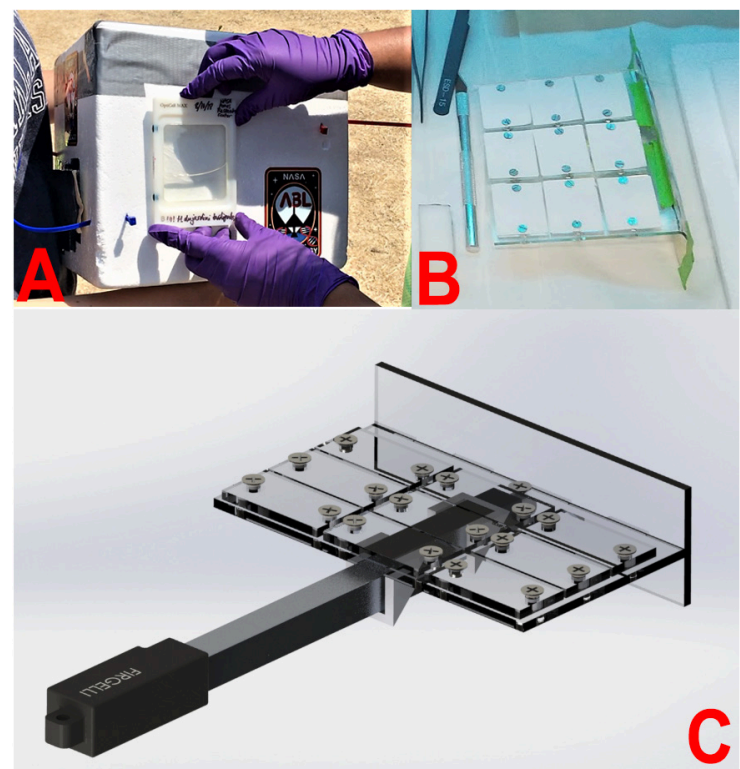

Figure 4. Photo of payload (A), BST with attached coupons and filter paper (B), BST assembly computer aided design (CAD) (C). calculation is made possible by publicly available software (https://github.com/Schm1tz1/arduino-ms5xxx). Time of day, altitude, pressure, temperature, and BST position were logged onto the microSD card. The data logging and test software are described in further detail on the SHAB GitHub repository (https://github.com/SLSTP-2018/shab) and are freely available for download.

\section{Payload Manufacturing}

The payloads were housed in Styrofoam boxes and were internally reinforced with lightweight foam core. Each flight included two BSTs, each with nine acrylic coupons, which were laser cut with a dimension of $5.08 \mathrm{~cm} \times 10.16 \mathrm{~cm} \times 0.375 \mathrm{~cm}$. The anterior faces of the coupons were covered with filter paper and screwed in place on the BSTs. The BSTs consisted of an acrylic platform $(18.58 \mathrm{~cm} \times 33.02 \mathrm{~cm} \times 0.3175 \mathrm{~cm})$ with pairs of holes for each coupon to be screwed into place. A protective shield was attached orthogonally to the exterior face of the BST to form a seal with the payload exterior when the BST was in the retracted position. The shield was created out of laser-cut acrylic $(33.02 \mathrm{~cm} \times 10.16 \mathrm{~cm} \times 0.3175 \mathrm{~cm})$. The linear actuator was fastened to the payload by rails, which were 3D-printed from the polylactic acid filament (LulzBot Taz 6). In our design, we positioned one linear actuator above the other in the payload box. To balance the payload's center of mass, BSTs extended in opposite directions. All of the 3D models for our custom-made parts can be downloaded from Grabcad (https://grabcad.com/library/biological-sample-traysbst-models-1).

Before launch, the BSTs, coupons, and filter paper were sterilized by $15 \mathrm{~min}$ of exposure to UV-C radiation in
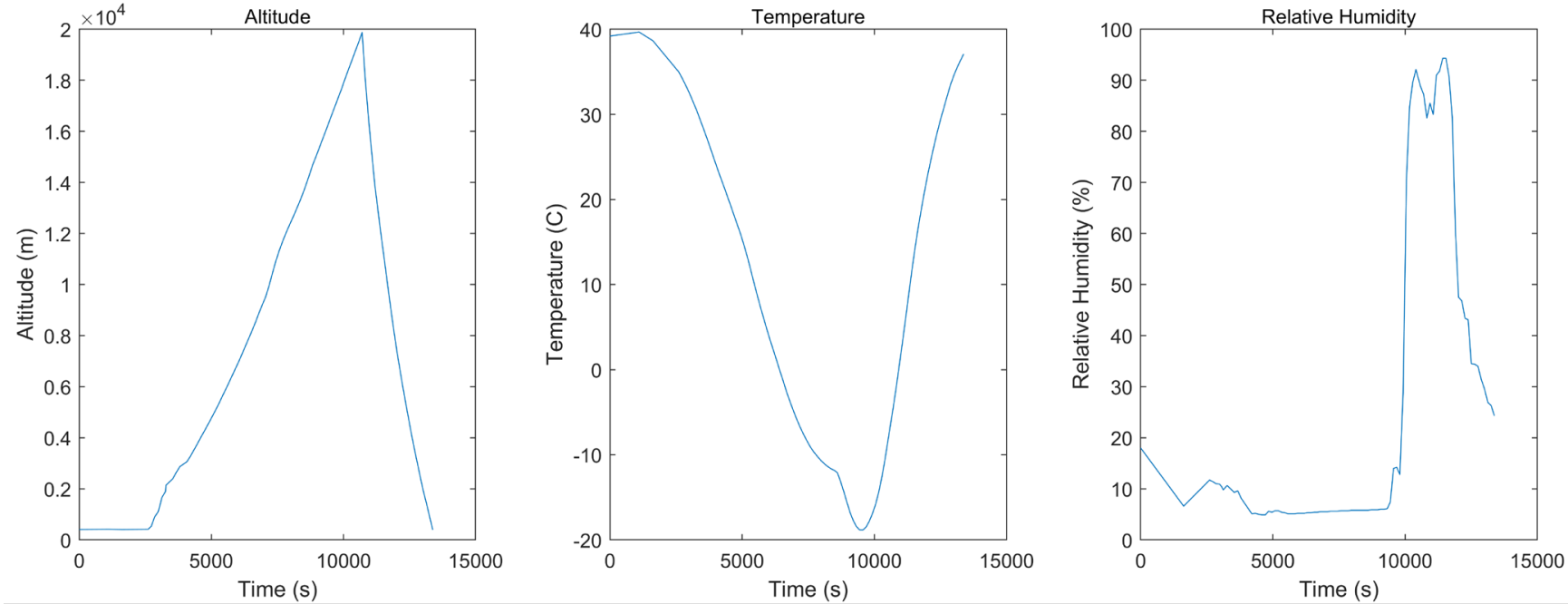

Figure 5. Altitude as measured by onboard pressure altimeter shows relative rates of payload ascent and descent. Temperature and relative humidity as recorded by sensors onboard SHAB-2 payload. 
a biological safety cabinet. Immediately after sterilization, the coupons were covered with sterile aluminum foil. The BSTs were mounted to the linear actuators, and coupons were uncovered moments before launch. Thus, the coupons remained shielded within the payload until it reached its target altitude, at which point the flight software triggered linear actuator extension and coupon exposure.

Flight hardware for one balloon was acquired and constructed for \$2,199.31, not including incidental retrieval expenses (i.e., food, gas, and lodging) with the payload weighing 1,645.7 g (full cost and mass budget in Appendix 1).

\section{Launch Protocol}

A $3.048 \mathrm{~m} \times 3.048 \mathrm{~m}$ woven poly tarp was placed on the flat ground underneath the area where the balloon was filled with helium. Payloads were attached to the Flightline with $0.3175 \mathrm{~cm}$ paracord, and the balloon was inflated with a $3 \mathrm{~cm}$ weather balloon inflator (High Altitude Science). The pressure of the helium tank decreased from 204 to $5.4 \mathrm{~atm}$ during inflation, as measured by the tank's valve gauge. As the balloon rose during inflation, three individuals steadied the balloon to ensure that it did not contact the ground. These individuals wore nitrile gloves to prevent oils and particles on their palms from degrading the balloon's integrity. The pre-launch neck-lift was measured by a digital fish scale (Dr. Meter). When the desired neck-lift, as calculated by the CUSF Burst Calculator, was achieved, the balloon was sealed with duct tape and plastic zip ties, attached to the flight line, and released. About $48 \mathrm{~h}$ before launch, we notified the local Federal Aviation Authority of our launch plans.

\section{DISCUSSION}

Given the accelerated time frame, low budget, and adaptability to a variety of investigations, our HAB payload is an excellent model for investigating the Mars-like environment in Earth's upper atmosphere. Simulating cold, dry, rarefied, and irradiated conditions observed at altitude are very difficult in a classroom or other low-cost setting. Therefore, we assert that the stratosphere is a useful analog fieldsite for those interested in studying microbial survivorship, multicellular organism response, or physical characteristics of a near-space environment. In addition, stratospheric conditions can also be used to test CubeSat instruments, hardware, and experiments that are intended for flight in low Earth orbit (LEO).

For the benefit of future HAB teams, we discuss the inherent variability in ballooning methods. It is essential to plan for the unpredictability of $\mathrm{HAB}$ flights and prepare for many contingencies, especially in terms of landing location and flight duration. Comprehensive preflight planning is necessary to ensure that there are protocols in place to deal with situations that may arise during payload recovery operations. To minimize science loss, we strongly recommend using the previously mentioned CUSF/ASTRA path predictor tools repeatedly in the days and hours leading up to the launch. Although prediction software is not perfect, it does constrain probable flight trajectories based on weather conditions. As shown in Figure 3, the CUSF software successfully determined the expected flight vector (azimuth from launch location) but was not accurate in predicting travel distance. HAB travel distance is a function of payload mass, wind speed, burst altitude, and parachute type. As an example of a prediction error, we suspect that our payload mass parameter used for SHAB2 trajectory prediction was an underestimate. SHAB-1 and SHAB-2 flew for 3 h 41 min and 2 h 57 min, respectively. Due to its large mass, SHAB-2 had a slow rate of ascent, allowing the balloon to travel further before reaching its burst altitude, thus causing it to overshoot the intended landing location. To minimize flight path variability, we strongly recommend taking advantage of the CUSF tool, which can take payload mass as an input to generate the neck-lift required to reach the desired burst altitude and landing location.

Experiments designed for LEO are quite distinct from traditional ground-based studies in terms of timeline, scope, and complexity. High-altitude ballooning exposes students to design constraints that are similar to those of research conducted in LEO; in that, both HAB and LEO payloads require extensive preflight planning and postflight analysis. The workflow required of $\mathrm{HAB}$ experiments affords students valuable experience in project management, addressing hardware issues as they arise, implementing engineering controls into experimental design, preparing for the possibility of science loss, and conducting biological research in the field-much like an LEO experiment.

\section{CONCLUSION}

We demonstrate that consumer-grade HABs can be used to access the upper troposphere and lower to middle stratosphere, where data can be collected for investigations pertaining to both the Earth's atmosphere and Mars-like conditions. In addition, we have demonstrated how HAB experimental design, construction, flight, and analysis can be accomplished on a low budget and accelerated time frame. This research vehicle is, therefore, suitable for a wide range of scientists, educators, and student researchers.

\section{DATA AVAILABILITY STATEMENT}

The data outlined in this report are available at https://github. com/tacaro/SHABprotocols for results of student-developed 
biological experiments; https://github.com/SLSTP-2018/shab for exposure sampling software; and https://grabcad.com/ library/biological-sample-trays-bst-models-1 for biological sampling tray designs.

\section{ACKNOWLEDGMENTS}

Funding for this study was provided by NASA Space Biology, whom we sincerely thank for their support throughout this project. We also sincerely thank Dr. David J. Smith of the NASA Ames Aerobiology Lab for providing essential lab resources and guidance. We would like to acknowledge Dr. Anthony Strawa, Dr. Sigrid Reinsch, Dr. Kevin Sato, Elizabeth Taylor, Sidney Sun, Matthew Lera, and the management of the NASA Ames Space Life Sciences Training Program (SLSTP) for making these flights possible. Special thanks to Daniel Mattox and Patrick Nicoll for their involvement in launch operations. We are grateful to Rebecca Sugrue and Dr. Thomas Kirchstetter at Lawrence Berkeley National Laboratory for the donation of two Aerosol Black Carbon Detectors (ABCDs) that were used during these flights. We sincerely thank US Forest Service Rangers Ethan Gicker, Charlie Hiles, Daniel Edward O'Halloran, and Kit Bailey for the recovery of SHAB-1 payload and their tireless efforts fighting the relentless California wildfires of 2018. We are grateful to ham operator Dana Myers (K6JQ) for volunteer assistance with radiolocation of SHAB-1. Finally, we would like to thank our two anonymous reviewers for their valuable feedback on this manuscript. Reference herein to any specific commercial product, process, or service by trade name, trademark, manufacturer, or otherwise does not necessarily constitute or imply its endorsement, recommendation, or favoring by the authors. The views and opinions expressed herein shall not be used for advertising or product endorsement purposes.

\section{AUTHOR DISCLOSURE STATEMENT}

No competing financial interests exist.

\section{AUTHORS' CONTRIBUTIONS}

$\mathrm{JM}, \mathrm{TC}, \mathrm{ET}, \mathrm{SV}$, and $\mathrm{AH}$ wrote the manuscript with input from all authors. AH and ET conceived of original circuits and computer programs described. ET and KC created CAD renderings of payload components. TC processed and mapped telemetry data. JR supervised, coordinated, and significantly contributed to manuscript revision. All authors contributed to the editing and proofreading of the manuscript. All authors contributed to the launch and recovery operations.

\section{REFERENCES}

Bryan N, Stewart M, Granger D, Guzik T, Christner B (2014) A method for sampling microbial aerosols using high altitude balloons. Journal Of Microbiological Methods 107, 161-168. doi: 10.1016/j. mimet.2014.10.007

Gurubaran S, Shanmugam M, Jawahar K, Emperumal K, Mahavarkar P, Buduru S (2017) A high-altitude balloon experiment to probe stratospheric electric fields from low latitudes. Annales Geophysicae 35(2), 189-201. doi: 10.5194/angeo-35-189-2017

Hofmann D, Harder J, Rolf S, Rosen J (1987) Balloon-borne observations of the development and vertical structure of the Antarctic ozone hole in 1986. Nature 326(6108), 59-62. doi: 10.1038/326059a0

Imshenetsky A, Lysenko S, Kazakov G (1976) Upper Boundary of the Biosphere. Applied and Environmental Microbiology 35(1), 1 - 5.

Kaplan D (1988) Environment of Mars. NASA STI/Recon Technical Report 89:14187.

Lawrence DJ, Fix S, Goldsten JO, Heuer SV, Hourani RS, Kerem S, Peplowski PN (2018) Near-space operation of compact Csl, CLYC, and $\mathrm{CeBr}_{3}$ sensors: Results from two high-altitude balloon flights. Nuclear Instruments and Methods in Physics Research Section A: Accelerators, Spectrometers, Detectors and Associated Equipment 905: 33-46

Pulschen A, de Araujo G, de Carvalho A, Cerini M, Fonseca L, Galante D, Rodrigues F (2018)

Survival of Extremophilic Yeasts in the Stratospheric Environment during Balloon Flights and in Laboratory Simulations. Applied And Environmental Microbiology 84(23). doi: 10.1128/aem.01942-18

Smith D, Griffin D, Schuerger A (2009) Stratospheric microbiology at $20 \mathrm{~km}$ over the Pacific Ocean. Aerobiologia 26(1), 35-46. doi: 10.1007/s10453-009-9141-7

Smith D, Griffin D, McPeters R, Ward P, Schuerger A (2011) Microbial survival in the stratosphere and implications for global dispersal. Aerobiologia 27(4), 319-332. doi: 10.1007/s10453-011-9203-5

Smith D (2013) Microbes in the Upper Atmosphere and Unique Opportunities for Astrobiology Research. Astrobiology 13(10), 981 990. doi: 10.1089/ast.2013.1074

Smith D, Sowa M (2017) Ballooning for Biologists. Gravitational and Space Research, 5(1), 52-73.

Yajima N, Izutsu N, Imamura T, Abe T (2004) Scientific Ballooning: Technology and Applications of Exploration Balloons Floating in the Stratosphere and the Atmospheres of Other Planets. New York: Springer. 
Appendix 1

\begin{tabular}{|c|c|c|c|c|c|c|c|}
\hline Item & Count & $\begin{array}{l}\text { Mass per item } \\
\text { (g) }\end{array}$ & $\begin{array}{l}\text { Total Mass } \\
\text { (g) }\end{array}$ & $\begin{array}{c}\text { Item cost } \\
\text { (w/o shipping) }\end{array}$ & Total & $\begin{array}{l}\text { With estimated } \\
\text { tax + shipping }\end{array}$ & Vendor \\
\hline Latex Balloon & 1 & 1500.0 & 1500.0 & $\$ 115.00$ & $\$ 115.00$ & $\$ 138.00$ & High Altitude Science \\
\hline Parachute & 1 & 190.0 & 190.0 & $\$ 85.00$ & $\$ 85.00$ & $\$ 102.00$ & High Altitude Science \\
\hline Paracord & 1 & 0.0 & 0.0 & $\$ 10.00$ & $\$ 10.00$ & $\$ 12.00$ & Paracord Planet \\
\hline Foil Tape & 1 & 0.0 & 0.0 & $\$ 3.00$ & $\$ 3.00$ & $\$ 3.60$ & Amazon \\
\hline Cardboard & 1 & 5.0 & 5.0 & $\$ 0.00$ & $\$ 0.00$ & $\$ 0.00$ & (salvaged recycling) \\
\hline \multicolumn{8}{|l|}{ Payload } \\
\hline Styrofoam Cooler & 1 & 50.0 & 50.0 & $\$ 30.00$ & $\$ 30.00$ & $\$ 36.00$ & Amazon \\
\hline Arduino Uno Starter Kit & 1 & 30.0 & 30.0 & $\$ 93.00$ & $\$ 93.00$ & $\$ 111.60$ & GigaParts \\
\hline Arduino battery & 1 & 52.0 & 52.0 & $\$ 15.00$ & $\$ 15.00$ & $\$ 18.00$ & Ada Fruit \\
\hline Altimeter & 1 & 0.5 & 0.5 & $\$ 30.00$ & $\$ 30.00$ & $\$ 36.00$ & Parallax \\
\hline $\begin{array}{l}\text { MicroTrak AIO HA w/ V6 } \\
\text { (batteries included) }\end{array}$ & 1 & 517.1 & 517.1 & $\$ 320.00$ & $\$ 320.00$ & $\$ 384.00$ & Byonics \\
\hline Linear Actuator & 2 & 56.0 & 112.0 & $\$ 70.00$ & $\$ 140.00$ & $\$ 168.00$ & Actuonix \\
\hline SD Card & 1 & 0.5 & 0.5 & $\$ 7.50$ & $\$ 7.50$ & $\$ 9.00$ & Adafruit \\
\hline $\begin{array}{c}\text { Coupons (32"x11" 1/16 } \\
\text { Acrylic) }\end{array}$ & 16 & 0.1 & 1.6 & $\$ 1.00$ & $\$ 16.00$ & $\$ 19.20$ & Lowe's Home Improvement \\
\hline Flat Head Machine Screws & 36 & 0.2 & 7.2 & $\$ 0.22$ & $\$ 7.92$ & $\$ 9.50$ & Home Depot \\
\hline 3D printed parts for sampler & 2 & 10.0 & 20.0 & $\$ 22.00$ & $\$ 44.00$ & $\$ 52.80$ & Lulzbot Taz 6 \\
\hline GoPro Hero 5 & 1 & 118.0 & 118.0 & $\$ 249.00$ & $\$ 249.00$ & $\$ 298.80$ & GoPro \\
\hline WingCam & 2 & 29.4 & 58.8 & $\$ 40.31$ & $\$ 80.62$ & $\$ 96.74$ & Hobby King \\
\hline \multicolumn{8}{|l|}{ Ground Equipment } \\
\hline RTL-SDR & 1 & NA & NA & $\$ 20.95$ & $\$ 20.95$ & $\$ 25.14$ & Amazon \\
\hline $\begin{array}{c}\text { Baofeng UV-5R Handheld } \\
\text { Transmitter }\end{array}$ & 2 & NA & NA & $\$ 34.99$ & $\$ 69.98$ & $\$ 83.98$ & Amazon \\
\hline Tarp & 1 & NA & NA & $\$ 7.99$ & $\$ 7.99$ & $\$ 9.59$ & Amazon \\
\hline Weather Balloon Inflator & 1 & NA & NA & $\$ 85.00$ & $\$ 85.00$ & $\$ 102.00$ & High Altitude Science \\
\hline Rental of Helium Tanks & 1 & NA & NA & $\$ 299.00$ & $\$ 299.00$ & $\$ 358.80$ & Party City (San Francisco) \\
\hline Latex Gloves & 1 & NA & NA & $\$ 9.85$ & $\$ 9.85$ & $\$ 11.82$ & Amazon \\
\hline Pelican Case & 1 & NA & NA & $\$ 48.00$ & $\$ 48.00$ & $\$ 57.60$ & Amazon \\
\hline Portable Soldering Kit & 1 & NA & NA & $\$ 26.00$ & $\$ 26.00$ & $\$ 31.20$ & Amazon \\
\hline Duct Tape & 1 & NA & NA & $\$ 14.00$ & $\$ 14.00$ & $\$ 16.80$ & Amazon \\
\hline Zip ties & 1 & NA & NA & $\$ 5.95$ & $\$ 5.95$ & $\$ 7.14$ & Amazon \\
\hline Total & & & 2662.70 & & $\$ 1,832.76$ & $\$ 2,199.31$ & \\
\hline $\begin{array}{l}\text { Total flight mass (minus } \\
1500 \mathrm{~g} \text { for balloon) }\end{array}$ & & & 1162.70 & & & & \\
\hline
\end{tabular}

